\title{
Normed quotient rings
}

\author{
Vakkas Ulucay ${ }^{1}$, Mehmet Sahin ${ }^{2}$ and Necati Olgun ${ }^{2}$ \\ ${ }^{1}$ Köklüce Neighbourhood, Araban, Gaziantep27310-Turkey \\ ${ }^{2}$ Department of Mathematics, Gaziantep University, Gaziantep27310-Turkey.
}

Received: 25 November 2016, Accepted: 17 June 2017

Published online: 7 February 2018.

\begin{abstract}
In this article, we introduce the notions of normed quotient ring, normed quotient subring, normed quotient ring homomorphism, normed quotient ring natural homomorphism and investigate some of their related properties.
\end{abstract}

Keywords: Ring, quotient ring, normed quotient ring, normed quotient subring.

\section{Introduction}

Ziembowski [27] exists a duo ring $R$ such that its classic al right ring of quotients $Q_{C l}^{r}(R)$ is left duo and not right duo. Building on this construction, we will construct a duo ring with classical right ring of quotients which is neither right nor left duo. For group rings the classical quotient ring has been studied by Herstein and Small [13], Passman [18, 19],Smith[21], and Smith[22], and the maximal quotient ring has been studied by Burgess [7]. Formanek [9] investigates the relationship of the maximal quotient rings of group rings, subgroup rings, and the centers of group rings. The object is to obtain for the maximal quotient ring analogues of theorems of Passman and M. Smith on the classical quotient ring. Ara [3] defined by $Z(R)$ the center of $R$ and by $Q(R)$ its maximal right quotient ring. Utumi[26] $R$ has always a unique maximal left quotient ring, and moreover the maximal left quotient ring of a total matrix ring of finite degree over $R$ is a total matrix ring of the same degree over the maximal left quotient ring of $R$. Johnson[16] it is assumed that each ring has a zero right singular ideal and given the quotient structure of a ring having a vanishing right and left singular ideal. Gary et al.[6].

For an arbitrary ring $R$ we completely characterize when $Q(R)$, the maximal right ring of quotients of $R$, is a direct product of indecomposable rings and when $Q(R)$ is a direct product of prime rings in terms of conditions on ideals of $R$. They also investigated the connections between the ideal structure of an arbitrary ring $R$ and the ideal structure of $Q(R)$. Francis [25] A ring $F$ has a semi simple maximal right quotient ring $Q$ if and only if $Z\left(R_{R}\right)=0$ and dim $R_{R}$ is finite, where a right $F$-module $M$ is of finite dimension if every direct sum of submodules of $M$ has only finitely many nonzero summands. Bavula [4] proved existence of the largest left quotient ring $Q_{1}(R)$, i.e. $Q_{1}(R)=S_{0}(R)^{(-1)} R$ where $S_{0}(R)$ is the largest left regular denominator set of $R$. Bavula [5] defined the largest strong left denominator set $T_{l}(R)$ of $R$, the largest strong left quotient $\operatorname{ring} Q_{l}^{s}(R):=T_{l}(R)^{(-1)} R$ of $R$ and the strong left localization radical $l_{R}^{s}$ of $R$, and to study their properties. Shilov [23] defined on decomposition of a commutative normed ring in direct sums of ideals. Gelfand et al. [10] defined commutative normed rings. Freundlich [8] introduced completely continuous elements of a normed ring.

Raikov [20] defined to the theory of normed rings with involution. Naimark et al. [17] defined normed rings. Shilov [24] defined analytic functions in a normed ring. Jarden [14] defined normed rings in 2011 and is studied norms $\|$.$\| of$ 
associative rings are generalizations of absolute values |.| of integral domains. Jung et al. [15] introduced quasi-commutative as a generalization of commutative rings. They also provide several sorts of examples by showing the relations between quasi-commutative rings and other ring properties which have roles in ring theory. Alvir [1] explored the compressed zero-divisor graph associated with quotient rings of unique factorization domains. Alizadeh [2] for $n \geq 3$, they showed that every local derivation from $M_{n}(R)$ into $M_{n}(M)$ is a derivation. Genc [11] given a construction of quotient gamma rings of prime gamma rings in the sense of Nobusawa and to study the some properties of quotient gamma rings of the prime gamma rings. In this paper, our work is organized as follows. We define normed quotient ring using normed spaces. In the section 2, well known results of some preliminaries are given. In section 3, we defined normed quotient ring, normed ideal, normed prime ideal, normed homomorphism, normed natural homomorphism and investigate some of their related properties.

\section{Preliminaries}

Definition 1. [17]. The norm $\|$.$\| , which is defined on rings, is a generalization of the absolute value |.| defined on integral$ domains. On rings, the standard rule $|x . y| \leq|x| \cdot|y|$ is replaced by $\|x . y\| \leq\|x\| .\|y\|$.

Definition 2. [14]. Let A be commutative ring with 1. An ultrametric absolute value of $A$ is a function $\|: A \rightarrow R$ satisfying the following conditions:

1. $|a| \geq 0$ and $|a|=0 \Leftrightarrow a=0$,

2.There exists $a \in A$ such that $0<|a|<1$,

3. $|a \cdot b|=|a| \cdot|b|$

4. $|a+b| \leq \max (|a|,|b|)$.

Definition 3. [12] Let $(A,+,$.$) be a ring and let I be an ideal of A$. Then the set

$$
A / I=\{I+a: a \in A\}
$$

is a ring under the following operations:

$$
\begin{gathered}
\text { Addition }:(I+a) \oplus(I+b)=I+(a+b) \\
\text { Multiplication: }(I+a) \otimes(I+b)=I+(a . b) .
\end{gathered}
$$

$(A / I, \oplus, \otimes)$ is called the quotient ring of $A$ with $I$.

Definition 4. [12]. Let I be an ideal of a ring A. The function $\Pi$, defined by

$$
\Pi: A \rightarrow A / I \text { and } \Pi(a)=I+a
$$

is an onto homomorphism. $\Pi$ is called the canonical homomorphism.

Definition 5. [12]. Let $f: A \rightarrow S$ be an onto ring homomorphism and let I be an ideal of $A$. If the kernel of $f$, Kerf $\subseteq$ I then

$$
A / I \cong S / f(I)
$$

If $f$ is not onto, then $A / I$ is isomorphic to a subring of $S / f(I)$. 


\section{Normed quotient rings}

Definition 6. Let $A / I$ be a ring. $A / I$ is called a normed ring if a non-negative real-valued function, namely a norm, $\|\cdot\|_{A / I}: A / I \rightarrow R$ is defined for all $\bar{x}, \bar{y} \in A / I$ which satisfies the following conditions:

(i) $\|\bar{x}\|_{A / I}=0 \Leftrightarrow x \in I$,

(ii) $\|\bar{x} \oplus \bar{y}\|_{A / I} \leq\|\bar{x}\|_{A / I}+\|\bar{y}\|_{A / I}$,

(iii) $\|-\bar{x}\|_{A / I}=\|-1 \otimes(\bar{x})\|_{A / I}=\|-1\| \cdot\|\bar{x}\|_{A / I}=\|\bar{x}\|_{A / I}$,

(iv) $\|\bar{x} \otimes \bar{y}\|_{A / I} \leq\|\bar{x}\|_{A / I} \cdot\|\bar{y}\|_{A / I}$,

Example 1. Let $Z / 2 Z=Z_{2}=\{\overline{0}, \overline{1}\}$. It is easy to show that the norm conditions.

(i) $\|\bar{x}\|_{A / I}=0 \Leftrightarrow x=0$,

(ii) $\|\overline{0} \oplus \overline{1}\|_{A / I} \leq\|\overline{0}\|_{A / I}+\|\overline{1}\|_{A / I}$,

(iii) $\|-\overline{1}\|_{A / I}=\|-1 \otimes(\overline{1})\|_{A / I}=\|-1\| \cdot\|\overline{1}\|_{A / I}=\|\overline{1}\|_{A / I}$,

(iv) $\|\overline{0} \otimes \overline{1}\|_{A / I} \leq\|\overline{0}\|_{A / I} \cdot\|\overline{1}\|_{A / I}$,

Therefore, the quotient rings satisfy the norm conditions.

Theorem 1. If $A$ is a normed ring with unity, then $A / I$ is a normed ring with unity whose unit is the mapping $\|\overline{1}\|_{A / I}$.

Proof. Let $\bar{x}, \bar{y} \in A / I, A / I=\left\{\begin{array}{c}x+I=\|\bar{x}\|, \text { if } x \notin I \\ x+0=0, \text { if } x \in I\end{array}\right.$ defined by $\|\bar{x}\|_{A / I}=\|x\|_{A}$

(i) $\|\bar{x}\|_{A / I}=0 \Leftrightarrow\|\bar{x}\|_{A / I}=\|x\|_{A}=0 \Leftrightarrow x=0$,

(ii) $\|-\bar{x}\|_{A / I}=\|-x\|_{A}=|-1|\|x\|_{A}=\|x\|_{A}=\|\bar{x}\|_{A / I}$,

(iii) $\|\bar{x} \oplus \bar{y}\|_{A / I}=\|x+y\|_{A} \leq\|x\|_{A}+\|y\|_{A}=\|\bar{x}\|_{A / I}+\|\bar{y}\|_{A / I}$,

(iv) $\|\bar{x} \otimes \bar{y}\|_{A / I}=\|x \cdot y\|_{A} \leq\|x\|_{A} \cdot\|y\|_{A}=\|\bar{x}\|_{A / I} \cdot\|\bar{y}\|_{A / I}$,

Theorem 2. Let $A$ be the normed ring. Then the mapping $f: A \rightarrow S$, defined by

$$
f(x)=\|x\|
$$

is an onto homomorphism. $f$ is called the normed ring homomorphism.

Proof. Let $x, y \in A$,

(i) $\|f(x)\|_{S}=0 \Leftrightarrow\|f(x)\|_{S}=\|x\|_{A}=0 \Leftrightarrow f(x)=f(0)=0$,

(ii) $\|-f(x)\|_{S}=\|f(-x)\|_{S}=\|-x\|_{A}=|-1|\|x\|_{A}=\|x\|_{A}=\|f(x)\|_{S}$,

(iii) $\|f(x \oplus y)\|_{S}=\|f(x) \oplus f(y)\|_{S}=\|x+y\|_{A} \leq\|x\|_{A}+\|y\|_{A}=\|f(x)\|_{S}+\|f(y)\|_{S}$,

(iv) $\|f(x \otimes y)\|_{S}=\|f(x) \otimes f(y)\|_{S}=\|x \cdot y\|_{A} \leq\|x\|_{A} \cdot\|y\|_{A}=\|f(x)\|\left\|_{S} \cdot\right\| f(y) \|_{S}$,

Theorem 3. Let $A$ be the normed ring and I be an ideal. Then the mapping $\|\cdot\|_{A / I}: A / I \rightarrow S \quad x+I=\bar{x} \in A / I$, defined by

$$
\|\bar{x}\|_{A / I}=\|x\|
$$

is an onto homomorphism. $\|\cdot\|_{A / I}$ is called the normed quotient ring homomorphism.

Proof. Let $\bar{x}, \bar{y} \in A / I$,

(i) $\|x\|_{S}=0 \Leftrightarrow\|x\|_{S}=\|x\|_{A / I}=0 \Leftrightarrow \bar{x}=0 \Leftrightarrow\|x\|=0 \Leftrightarrow x=0$,

(ii) $\|-\bar{x}\|_{A / I}=\|-x\|_{S}=|-1|\|x\|_{S}=\|x\|_{A}=\|x\|_{S}=\|\bar{x}\|_{A / I}$,

(iii) $\|\bar{x} \oplus \bar{y}\|_{A / I}=\|x+y\|_{S} \leq\|x\|_{S}+\|y\|_{S}=\|\bar{x}\|_{A / I}+\|\bar{y}\|_{A / I}$, 
(iv) $\|\bar{x} \otimes \bar{y}\|_{A / I}=\|x \cdot y\|_{S} \leq\|x\|_{S} \cdot\|y\|_{S}=\|\bar{x}\|_{A / I} \cdot\|\bar{y}\|_{A / I}$,

Theorem 4. (Decomposition of Normed Homomorphism) Let $f: A \rightarrow S$ be a normed ring homomorphism and let $I=$ Kerf. Then, there exists a unique normed homomorphism is an onto homomorphism. $f^{\prime}: A / I \rightarrow f(A)$ such that $f=i f^{\prime} \phi$ where $\phi: A \rightarrow A / I$ is the normed natural homomorphism and $i: f(A) \rightarrow S$ is the (one-to-one) injection mapping.

Proof.

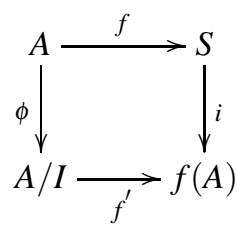

be a commutative diagram. It is enough to define the normed homomorphism $f^{\prime}: A / I \rightarrow f(A), \forall r \in A \quad f^{\prime}(\bar{r})=f(r)$. First, we show that $f^{\prime}$ is well-defined.

$$
\begin{aligned}
\bar{r}=\bar{r}^{\prime} & \Rightarrow r-r^{\prime} \in I=\operatorname{Ker} f \\
& \Rightarrow\|r\|_{A}=\left\|r^{\prime}\right\|_{A} \\
& \Rightarrow f(r)=f\left(r^{\prime}\right) \\
& \Rightarrow f^{\prime}(\bar{r})=f^{\prime}\left(\bar{r}^{\prime}\right)
\end{aligned}
$$

So, $f^{\prime}$ is well-defined. $f=i f^{\prime} \phi$ and that $f^{\prime}$ is an onto normed homomorphism is trivial. Now, we show the uniqueness of $f^{\prime}$.

Let $g: A / I \rightarrow f(A)$, be another normed homomorphism such that $f=i f^{\prime} \phi$. Then, $\forall r \in A$,

$$
\begin{aligned}
f^{\prime}(\bar{r}) & =f(r)=i g(\phi(r)) \\
& =i g(\bar{r})=g(\bar{r})
\end{aligned}
$$

Thus $f^{\prime}=g$ and $f^{\prime}$ is unique.

Theorem 5. Let $A$ be a normed ring and I be an ideal of A. Let

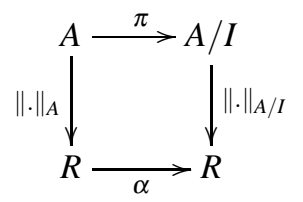

be a commutative diagram. Then the mapping $\alpha: \mathbb{R} \rightarrow \mathbb{R}$ defined by

$$
\alpha\left(\|x\|_{A}\right)= \begin{cases}\alpha\left(\|x\|_{A}\right)=\|\bar{x}\|_{A / I}, & x \in A \\ \alpha\left(\|x\|_{A}\right)=x, & x \notin A\end{cases}
$$

is an onto homomorphism whose kernel is I. This normed homomorphism is called the normed natural homomorphism from $A$ onto $A / I$.

Proof. Let $\bar{x} \in A / I$. As $\alpha\left(\|x\|_{A}\right)=\|\bar{x}\|_{A / I}, \alpha$ is onto. Also $\forall x, y \in A$,

$$
\alpha\left(\|x+y\|_{A}\right)=\|\bar{x} \oplus \bar{y}\|_{A / I} \leq\|\bar{x}\|_{A / I}+\|\bar{y}\|_{A / I}=\alpha\left(\|x\|_{A}\right)+\alpha\left(\|y\|_{A}\right)
$$

and

$$
\alpha\left(\|x . y\|_{A}\right)=\|\bar{x} \otimes \bar{y}\|_{A / I} \leq\|\bar{x}\|_{A / I} \cdot\|\bar{y}\|_{A / I}=\alpha\left(\|x\|_{A}\right) \cdot \alpha\left(\|y\|_{A}\right)
$$


This shows that $\alpha$ is a normed homomorphism. Also by

$$
\bar{k} \in \operatorname{Ker}(\alpha) \Leftrightarrow \alpha\left(\|k\|_{A}\right)=\|\bar{k}\|_{A / I}=0=I \Leftrightarrow k=I \Leftrightarrow k \in I,
$$

we have $\operatorname{Ker}(\alpha)=I$.

Example 2. Let $A$ be a normed ring and $I$ be an ideal of $A$. Show that $A / I$ is a normed commutative quotient ring if and only if for all $x, y \in A, x . y-y . x \in I$. Solution:

$$
\begin{aligned}
A / I \text { is commutative } & \Leftrightarrow \forall x, y \in A,\|\bar{x}\|_{A / I} \cdot\|\bar{y}\|_{A / I}=\|\bar{y}\|_{A / I} \cdot\|\bar{x}\|_{A / I} \\
& \Leftrightarrow \forall x, y \in A,\|\bar{x} \otimes \bar{y}\|_{A / I}=\|\bar{y} \otimes \bar{y}\|_{A / I} \\
& \Leftrightarrow \forall x, y \in A,\|\bar{x} \otimes \bar{y}\|_{A / I}-\|\bar{y} \otimes \bar{x}\|_{A / I}=0 \\
& \Leftrightarrow \forall x, y \in A,\|\bar{x} \otimes \bar{y} \ominus \bar{y} \otimes \bar{x}\|_{A / I}=0 \\
& \Leftrightarrow \forall x, y \in A, x . y-y \cdot x \in I .
\end{aligned}
$$

Theorem 6. Let $f: A \rightarrow S$ be an onto normed ring homomorphism and I be a (normed) ideal of $A$. If Kerf $\subseteq$ I, then

$$
A / I \cong S / f(I)
$$

If $f$ is not onto, then $A / I$ is isomorphic to a normed subring of $S / f(I)$.

Proof. Let $\phi: S \rightarrow S / I$ be the natural normed homomorphism. Take the normed homomorphism $g: A \rightarrow S / f(I)$ such that $g=\phi f$. Then $\forall r \in A$,

$$
g(r)=\phi(f(r))=\|\bar{f}(r)\|_{A / I}
$$

$g$ is the composition of two onto normed homomorphisms and hence is onto.

$$
\begin{aligned}
\text { Kerg } & =\{r \in A: \bar{f}(r)=f(I)\} \\
& =\{r \in A: f(r) \in f(I)\} \\
& =f^{-1}(f(I)) .
\end{aligned}
$$

Clearly, $I \subseteq f^{-1}(f(I))$. On the other hand as $\operatorname{Ker} f \subseteq I$,

$$
\begin{aligned}
x \in f^{-1}(f(I)) \Rightarrow & f(x) \in f(I) \\
& \Rightarrow \text { there exists } a \in I \text { such that }\|x\|_{A}=\|a\|_{A} \\
& \Rightarrow\|x-a\|_{A} \\
& \Rightarrow x-a \in \operatorname{Ker} f \subseteq I \\
& \Rightarrow x \in I .
\end{aligned}
$$

This shows that $f^{-1}(f(I)) \subseteq I$. Therefore, Kerg $=f^{-1}(f(I))=I$. By the Normed Homomorphism Theorem, we have

$$
A / I \cong S / f(I)
$$

If $f$ is not onto, it is easy to see that $A / I$ is isomorphic to a normed subring of $S / f(I)$.

Theorem 7. Let $A$ be a commutative normed ring with unity and $I \subseteq A$ be an ideal. I is a normed prime ideal $\Leftrightarrow A / I$ is an integral domain. 
Proof. $(\Rightarrow)$ Let $I$ be the prime ideal. As $I \neq A, A / I$ has at least two elements. Take $\bar{x}, \bar{y} \in A / I$ and recall that $I$ is the zero of $A / I$.

$$
\begin{array}{rl}
\bar{x} \cdot \bar{y}=I & x \cdot y+I=I \\
& \Rightarrow(x+I)(y+I)=I \\
& \Rightarrow x \cdot y \in I \\
& \Rightarrow x \in I \text { or } y \in I \text { (since is a prime ideal) } \\
& \Rightarrow \bar{x}=I \text { or } \bar{y}=I
\end{array}
$$

Thus, $A / I$ doesn't have any zero divisors. As $A$ is commutative and has a unity, so does $A / I$. This concludes that $A / I$ is an integral domain.

$(\Leftarrow)$ Let $A / I$ be an integral domain. Then, $A / I$ has at least two elements and hence $I \neq A$.

Let $x, y \in A$,

$$
\begin{aligned}
x . y \in I & \Rightarrow\|\bar{x} \otimes \bar{y}\|_{A / I} \\
& \Rightarrow\|\bar{x}\|_{A / I} \cdot\|\bar{y}\|_{A / I} \\
& \Rightarrow x \cdot y \in I \\
& \Rightarrow x=I \text { or } y=I \text { (since } A / I \text { is an integral domain) } \\
& \Rightarrow x \in I \text { or } y \in I
\end{aligned}
$$

showing that $I$ is a normed prime ideal.

\section{Conclusion}

In this paper, we examine the algebraic properties of normed quotient ring in ring structures. Some related notions, e.g the homomorphism on normed quotient ring, normed quotient subring, normed quotient ideal are proposed here. This concept will bring a new opportunity in research and development of normed ring theory.

\section{Competing interests}

The authors declare that they have no competing interests.

\section{Authors' contributions}

All authors have contributed to all parts of the article. All authors read and approved the final manuscript.

\section{References}

[1] Alvir, R. Zero-Divisor Graphs of Quotient Rings. (2015).arXiv preprint arXiv:1508.02432.

[2] Alizadeh, R., Bitarafan, M. J. Local derivations of full matrix rings. Acta Mathematica Hungarica, 145(2),(2015), $433-439$.

[3] Ara, P. Centers of maximal quotient rings. Archiv der Mathematik,(1988), 50(4), 342-347.

[4] Bavula, V. V. The largest left quotient ring of a ring. (2011), arXiv preprint arXiv :1101. 5107.

[5] Bavula, V. V. The largest strong left quotient ring of a ring. Journal of Algebra,(2015), 439, 1-32.

[6] Birkenmeier, G. F., Park, J. K., Rizvi, S. T. The structure of rings of quotients. Journal of Algebra, (2009), 321(9),2545-2566.

[7] Burgess,W., Rings of quotients of group rings, Canad. J. Math.,(1969), 2 1, 865-875.

[8] Freundlich, M. ,'Completely continuous elements of a normed ring.”Duke Math. J 16.1 (1949): 949. 
[9] Formanek, E. Maximal quotient rings of group rings. Pacific Journal of Mathematics, (1974), 53(1), 109-116.

[10] Gelfand,I.M., D.A. Raikov, and G. E. Silov. Commutative normed rings. American Mathematical Society, 1957.

[11] Genc, A. On Quotient Rings Of Prime Gamma Rings and Some Properties. Ege University Journal Of The Faculty Of Science,(2015), 39(2), 11-22.

[12] Gilbert, W.J., Modern Algebra with Applications.A Wiley Interscience Pubc. Inc. John Wiley and Sons. Inc. (1976).

[13] Herstein,I. N. and L. Small, Rings of quotients of group algebras, J. Algebra, 19 (1971), 153-155.

[14] Jarden,M. , ’Normed Rings.” Algebraic Patching. Springer Berlin Heidelberg, 2011, 10-30.

[15] Jung, D. W., Kim, B. O., Kim, H. K., Lee, Y., Nam, S. B., Ryu, S. J., Yun, S. J. On Quasi-Commutative Rings. Journal of the Korean Mathematical Society,(2016), 53(2), 475-488.

[16] Johnson, R. E. Quotient rings of rings with zero singular ideal. Pacific J. Math,(1961), 11(4), 1385-1392.

[17] Naimark,M.A., Normed rings. P. Noordhoff, 1964.

[18] Passman,D. S., Infinite Group Rings, Dekker, New York, 1971.

[19] Passman,D. S., On the ring of quotients of a group ring, Proc. Amer. Math. Soc, 33 (1972), 221-225.

[20] Raikov, D. A. "To the theory of normed rings with involution.” CR (Doklady) Acad. Sci. URSS (NS). Vol.54.1946.

[21] Smith,M., Group algebras, J. Algebra, 18 (1971), 477-499.

[22] Smith,P. F., Localization in group rings, Proc. London Math. Soc, (3) 22 (1971), 69-90.

[23] Shilov,G.E., "On decomposition of a commutative normed ring in a direct sums of ideals. " Matematicheskii Sbornik 74.2 (1953): 353-364.

[24] Shilov,G.E. ”Analytic functions in a normed ring.” Uspekhi Matematicheskikh Nauk 15.3 (1960): 181-183.

[25] Sandomierski, F. L. Semisimple maximal quotient rings. Transactions of the American Mathematical Society,(1967), 128(1), 112120.

[26] Utumi, Y. On quotient rings.(1956).

[27] Ziembowski, M. On classical rings of quotients of duo rings. Journal of Pure and Applied Algebra,(2014), 218(5), 919-924. 